\title{
Volumineuse lithiase urinaire vésicale évacuée par voies naturelles
}

\section{Spontaneous Evacuation of Bulky Bladder Urolithiasis}

\section{F. Balen $\cdot$ B. Ribere $\cdot$ M. Bastouil}

Reçu le 23 octobre 2018; accepté le 24 janvier 2019

(C) SFMU et Lavoisier SAS 2019

Un homme de 66 ans est retrouvé confus auprès de sa voiture dans le parking de sa résidence par des voisins qui contactent le centre 15. Les paramètres relevés par les pompiers à l'arrivée sont les suivants : tension artérielle à 120/65 $\mathrm{mmHg}$, une fréquence cardiaque à 82 battements/minute et une $\mathrm{SpO}_{2}$ à $95 \%$ en air ambiant. À l'arrivée aux urgences, le malade est apyrétique et présente une glycémie capillaire à $1,13 \mathrm{~g} / \mathrm{l}$. L'examen clinique initial permet de constater d'emblée une urétrorragie faisant découvrir une lithiase à l'abouchement de l'urètre. Cette dernière est retirée par pression de la verge de la base à la distalité et se révèle être volumineuse (Fig. 1). La douleur cède suite à ce geste d'évacuation, et le patient rapporte alors une chirurgie de résection transurétrale de prostate (RTUP) le mois précédent. L'échographie de l'appareil urinaire réalisée dans les suites ne révèle pas de dilatation des cavités pyélocalicielles ni d'autres images lithiasiques rénale ou vésicale. Le patient est hospitalisé en service de posturgence du fait d'une infection urinaire consécutive à l'obstacle, avant de retourner à son domicile. La prévalence de la lithiase urinaire est estimée aux alentours de $10 \%$ dans la population générale occidentale [1]. Elle est surtout représentée par la lithiase du haut appareil urinaire. La lithiase vésicale est quant à elle paucisymptomatique et son épidémiologie mal connue. Sa prévalence est estimée à $10 \%$ chez les patients porteurs d'une hypertrophie bénigne de prostate bénéficiant de RTUP [2]. Aussi, bien que cette pratique ne fasse pas stricto sensu l'objet de recommandations par les sociétés savantes d'urologie, il est classique de réaliser une évacuation des lithiases vésicales concomitante d'un geste de RTUP. En conclusion, l'évacuation d'une lithiase vésicale

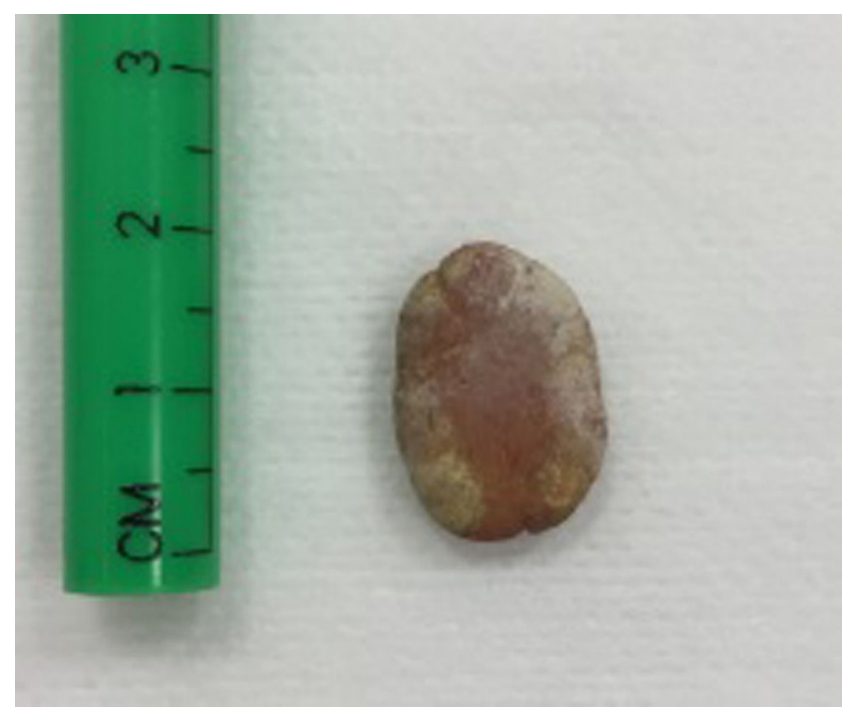

Fig. 1 Lithiase urinaire

par voies naturelles est une complication possible d'une RTUP, dans le cas où cette dernière n'est pas retirée en peropératoire.

\section{Références}

1. Daudon M, Traxer O, Lechevallier E, et al (2008) Epidemiology of urolithiasis. Prog Urol 18:802-14

2. Kim JW, Oh MM, Park HS, et al (2014) Intravesical prostatic protrusion is a risk factor for bladder stone in patients with benign prostatic hyperplasia. Urology 84:1026-9

\footnotetext{
F. Balen $(\bowtie) \cdot$ B. Ribere $\cdot$ M. Bastouil

Pôle de médecine d'urgence/Samu, CHU de Toulouse, place du Docteur-Baylac, TSA 40031,

F-31059 Toulouse cedex 09, France

e-mail : balen.f@chu-toulouse.fr

F. Balen

Université Toulouse-III-Paul-Sabatier,

118 , route de Narbonne,

F-31062 Toulouse cedex 09, France
} 